\title{
Predictive value of psychological assessment at five years of age in the long-term follow-up of very preterm children
}

\section{Lind, Annika}

2020-04-02

Lind, A , Nyman , A , Lehtonen , L \& Haataja , L 2020 , ' Predictive value of psychological assessment at five years of age in the long-term follow-up of very preterm children ' , Child Neuropsychology , vol. 26 , no. 3 , pp. 312-323 . https://doi.org/10.1080/09297049.2019.1674267

http://hdl.handle.net/10138/319793

https://doi.org/10.1080/09297049.2019.1674267

acceptedVersion

Downloaded from Helda, University of Helsinki institutional repository.

This is an electronic reprint of the original article.

This reprint may differ from the original in pagination and typographic detail.

Please cite the original version. 
Predictive Value of Psychological Assessment at Five Years of Age in the Long-Term Follow-Up of Very Preterm Children

PhD Annika Lind ${ }^{1,2}$, LicA Anna Nyman ${ }^{1}$, Prof Liisa Lehtonen ${ }^{3}$ and Prof Leena Haataja ${ }^{4}$ on behalf of the PIPARI Study Group

${ }^{1}$ Department of Psychology, University of Turku, Turku, Finland

${ }^{2}$ Turku Institute for Advanced Studies (TIAS), University of Turku, Turku, Finland

${ }^{3}$ Department of Pediatrics, University of Turku and Turku University Hospital, Turku,

Finland

${ }^{4}$ Children's Hospital, Pediatric Research Center, University of Helsinki and Helsinki

University Hospital, Helsinki, Finland

Corresponding author: Annika Lind, Department of Psychology, University of Turku, 20014

Turun yliopisto, Finland (annika.lind@utu.fi)

Word count: 3611

Funding: This work was supported by Turku Institute for Advanced Studies 


\section{Abstract}

The aims of this study were 1) to assess the predictive value of psychological assessment at five years of age on the need for educational support in very preterm children, and 2) to report the neuropsychological profile of very preterm children at eleven years of age and risk factors for poorer neuropsychological functions. A cohort of 167 very preterm children was included (birth weight $\leq 1500 \mathrm{~g}$ and/or gestational age $<32$ weeks). Mean birth weight was $1116 \mathrm{~g}$ (SD 311, min 400, $\max 2120$ ) and mean gestational age 28.9 weeks (SD 2.7, min 23.0, $\max 35.9)$. At five years of age, intellectual functioning was assessed with Wechsler Preschool and Primary Scale of Intelligence-Revised and neuropsychological performance with NEPSY II. At eleven years of age, neuropsychological functions were assessed using NEPSY II and data on educational support services collected using a questionnaire. Lower full-scale intelligence quotient and poorer performance in subtests inhibition, comprehension of instructions, memory for designs, visuomotor precision and design copying at five years of age were associated with a need for educational support at eleven years of age.

Neuropsychological performance at eleven years of age was overall within the average range but below the mean, with the poorest performance in tasks assessing visual memory and visuospatial functions. The results offer a novel perspective to timing and measures of follow-up of very preterm children, since they show that need for long-term educational support can be identified at five years of age. The findings also highlight the clinical value of psychological assessments including evaluation of both intellectual functioning and neuropsychological performance, covering detailed information about non-verbal functions, in the follow-up of very preterm children up to eleven years of age.

Keywords: very preterm, follow-up, developmental trajectory 


\section{Introduction}

Impairments in neuropsychological functions have been related to prematurity ${ }^{1-5}$ as well as to learning disabilities ${ }^{6}$. Neuropsychological functions include specific cognitive domains such as visual perceptual and visual motor skills, memory, language functions and executive functions. They correspond to intellectual functioning, but the association is not as clear in preterm children as in term-born children ${ }^{5}$, and neuropsychological functions can also be weaker in preterm children with average intellectual functioning ${ }^{4}$. In addition to poorer overall neuropsychological performance, their neuropsychological profiles appear more divergent compared to those of full term controls ${ }^{5}$.

Identifying deviating development in preterm children as early as possible is important since it enables targeted interventions and developmental support in order to strengthen skills, prevent the intensification of difficulties and the development of possible secondary problems. As preterm children evidently have poorer cognitive outcomes than children born at term ${ }^{7}$, systematic follow-up of preterm children is required. However, the most informative assessments and time points have not been agreed upon. The interval, length and focus of follow-up vary between countries as well as between hospitals, and there are diverging findings about the predictive value of assessments at different ages ${ }^{1,8-19}$.

The stability of intellectual functioning between two and five years of age $\mathrm{e}^{20}$ as well as between five and eleven years of age ${ }^{21}$ has previously shown to be good in longitudinal follow-up of preterm children. It has also been shown that preterm children may experience neuropsychological difficulties at five years of age in spite of average intellectual functioning ${ }^{4}$. Consequently there is a need to expand the evaluation of the follow-up. Further, 
very preterm children with age-appropriate educational abilities and without significant cognitive impairment have been shown to need more educational support services in school at eleven years of age than their peers born at term ${ }^{22}$.

To this date, there is no agreement or general practice regarding psychological assessments in the follow-up of very preterm children. Also, to our knowledge, there are no previous studies on associations between psychological assessment at five years of age and later need for educational support services. Thus, in order to increase knowledge about the clinical value of psychological assessment at five and eleven years of age in very preterm children, the specific aims of the present study were 1) to assess the predictive value of intellectual functioning and neuropsychological profile in very preterm children at five years of age in relation to educational support including studying on a grade below own age group, a need of full-time or part-time special education and/or a personal assistant at eleven years of age, and 2) to report the neuropsychological profile and risk factors for weaker neuropsychological functions at eleven years of age in the study cohort. We hypothesized that performance at five years of age relates to later need for educational support, and that neuropsychological difficulties will still be detected at eleven years of age. 


\section{Methods}

\section{Participants}

This study is part of the multidisciplinary PIPARI project (Development and Functioning of Very Low Birth Weight Infants from Infancy to School Age). All very low birth weight ( $\leq$ $1500 \mathrm{~g}$ ) infants born at Turku University Hospital in Finland between 2001 and 2006 who lived in the hospital catchment area and whose parents spoke and understood written Finnish or Swedish were eligible. From the beginning of 2004, the inclusion criteria were expanded to include all infants born $<32$ weeks of gestation, regardless of their birthweight. After excluding 12 infants with major congenital anomalies or syndromes or chromosomal anomalies, 228 infants were eligible and the parents of 219 infants chose to participate in the main PIPARI study. Of those, 167 (73\% of 228) Finnish-speaking children participated in the psychological assessment at eleven years of age. Data concerning both the psychological assessment at five years of age and educational support at eleven years of age were available for 150 Finnish-speaking children. Informed consent was obtained from the parents, and at eleven years of age, the children also gave their own written informed consent after receiving written information. The study has been approved by the Ethical Committee of the Hospital District of Southwest Finland.

\section{Psychological assessment at five years of age}

The time point of five years of age was chosen because neuropsychological functions can be studied more distinctly than at earlier ages. In addition, the development can be supported before entering school (at seven years of age in Finland) if risk factors for learning difficulties are identified. At five years of age $(M=5$ years 1 month, $S D=1$ month $)$, the children were assessed by a psychologist blinded to perinatal history. Intellectual functioning was assessed using the Finnish version of Wechsler Preschool and Primary Scale of 
Intelligence- Revised $^{23}$ test and the full-scale intelligence quotient (FSIQ; $M=100, S D=15$ ) was estimated as described in Lind et al. ${ }^{4}$. Neuropsychological performance was assessed using age appropriate subtests from the standardization edition of the Finnish NEPSY II ${ }^{24,25}$. Attention and executive functioning were evaluated with subtests visual attention, assessing selective visual attention; auditory attention, assessing selective auditory attention and the ability to sustain it; and inhibition, assessing the ability to inhibit automatic responses. Language functions were evaluated with subtests speeded naming, assessing rapid semantic access to and production of words; comprehension of instructions, assessing the ability to receive and process oral instructions of increasing syntactic complexity; and phonological processing, assessing phonemic awareness. Memory was evaluated with subtests memory for designs, assessing spatial memory for novel visual material; narrative memory, assessing memory for logical verbal material under free and cued recall; and word list interference assessing verbal working memory. In addition, visuomotor and visuospatial functions were evaluated with subtests visuomotor precision, assessing graphomotor speed and accuracy and design copying assessing motor and visual-perceptual skills associated with the ability to copy two-dimensional geometric figures. The standard scores $(\mathrm{M}=10, \mathrm{SD}=3)$ were based on the results of a control group of 149 healthy term-born children participating in the PIPARI study. The subtests, the differences between the standardization and the final edition as well as the control group have been described in Lind et al. ${ }^{26}$.

\section{Psychological assessment at eleven years of age}

The age point of eleven years was chosen because the need for educational support should have appeared and been recognized by this age through the increasing academic demands. Identification of specific neuropsychological impairments before entering junior high school is also of importance. The children participated in a psychological examination the year they 
turned eleven ( $\mathrm{M}=11$ years 2 months, $\mathrm{SD}=3$ months). The psychologist was blinded to the children's perinatal history and to the results of the psychological assessment at five years of age. The testing procedure has been described in Nyman et al. ${ }^{21}$. Neuropsychological functioning was evaluated using age appropriate subtests from the NEPSY II $^{24,25}$. The above described subtests comprehension of instructions, memory for designs, narrative memory, visuomotor precision and design copying were used. In addition, the subtests word generation, assessing verbal productivity through the ability to generate words within specific categories and arrows, assessing the ability to judge line orientation, were included. Scores were based on age appropriate norms from the Finnish standardization $(M=10, S D=3)$. A standard score of eight or above was considered as average, of six or seven as slightly below average, and of five or below as significantly below average.

Data on educational support services - the child being 1) one or more grades below their own age group (yes/no), 2) in full-time special education (yes/no), 3) in part-time special education (yes/no), and 4) a personal assistant at school (yes/no) - were received from teachers using a questionnaire developed for the PIPARI project ${ }^{22}$. In many countries, children can enter school one year later if school readiness is suspected to be inadequate or they can repeat a class if they have difficulties with basic academic skills. Part-time special education is meant as support in a specific area, such as literacy or mathematics, and full-time special education is intended for children with a long-term need of support and includes mainly individualized education plans in one or several subjects. Full-time special education is available at special schools or in special classes, or it can be integrated into mainstream classes. Personal assistants are provided for instance for children with neurosensory impairments as the only measure or along with other support services. Overall, children can 
receive one or several support services. The need for support services is evaluated multiprofessionally. The categories are analysed separately in this study because of their differences in intensity and content. Separate analyses also ease comparison with other studies reporting outcome from different schooling systems as well as the repeatability of this study.

\section{Data analysis}

Statistical analyses were performed by a statistician. Drop out analysis was performed using two sample t-test, $\chi 2$-test or Fisher's exact test, as appropriate; Children who participated in the psychological assessment at eleven years of age $(n=167)$ were compared to those who had withdrawn from the PIPARI study after recruitment $(n=52)$ regarding the variables in Table 1. The impacts of parental education, sex, gestational age, antenatal growth restriction (birth weight z score), postnatal corticosteroids, neonatal illness (chronic lung disease, sepsis or meningitis and/or intestinal perforation) and brain magnetic resonance imaging (MRI) findings at term equivalent age on NEPSY II scores at eleven years of age were assessed using multiple regression analysis. Education was categorized based on education levels in Finland: $<9$ years, 9-12 years and $>12$ years. The brain MRI was performed with an open 0.23-T Outlook GP (Philips Medical, Vantaa, Finland) for infants born between 2001 and April 2004 and with a 1.5-T Philips Intera (Philips Medical Systems, Best, The Netherlands) for infants born thereafter. The imaging took place during postprandial sleep without pharmacological sedation or anesthesia and ear protection was used. The MRI findings were categorized as normal, minor or major as follows: normal findings consisted of normal brain anatomy (cortex, basal ganglia and thalami, posterior limb of internal capsule, white matter, germinal matrix, corpus callosum, and posterior fossa structures), width of extracerebral space of $<5 \mathrm{~mm}$, ventricular/brain ratio of $<0.35$, and no ventriculitis; minor pathologies consisted of consequences from intraventricular hemorrhages (grades 1 and 2), 
caudothalamic cysts, a width of the extracerebral space of $5 \mathrm{~mm}$, and a ventricular/brain ratio of 0.35 ; and major pathologies consisted of consequences from intraventricular hemorrhages (grades 3 and 4), an injury in the cortex, basal ganglia, thalamus, internal capsule, corpus callosum, cerebellum, or white matter, as well as increased width of extracerebral space by > $5 \mathrm{~mm}$, a ventricular/brain ratio of $>0.35$, ventriculitis, or other major brain pathologies (infarctions). Since the educational variables were dichotomous, the predictive value of test scores at five years of age on the need for educational support services at eleven years of age was assessed using univariate logistic regression analysis. The analyses were repeated adjusting for gender, dichotomous MRI (major/not major) and paternal education ( $\leq 12$ years I $>12$ years). Personal assistant could not be included in the analyses adjusted for background variables due to small number of observations in this support category. For the statistical analyses, a 9.4 version of SAS Institute Inc. (Cary, NC, USA) for Windows was used, and pvalues $<0.01$ were considered statistically significant. 


\section{Results}

At eleven years of age, 26 (16\%) very preterm children had neurodevelopmental impairments (14 had FSIQ < 70, four had cerebral palsy, three had severe hearing impairment, and five had both FSIQ $<70$ and cerebral palsy). Mean FSIQ at eleven years of age was 87.6 (SD 17.6). Background data on the very preterm children and the drop out children are presented in Table 1.

Thirty-two (21\%) of the children were one or more grades below their age group, full-time special education was received by $20(14 \%)$ and part-time special education by $29(20 \%)$, and three $(2 \%)$ children had a personal assistant at eleven years of age. Thirty-five (23\%) children received one support service, $21(14 \%)$ two or more and a total of $56(37 \%)$ children received some support service. Test scores at five years of age are presented in Table $2 \mathrm{a}$ and univariate associations between test scores at five years of age and educational support at eleven years of age in Table $2 b$. The need for various types of educational support services was predicted by lower FSIQ and by poorer scores in subtests inhibition, comprehension of instructions, memory for designs, visuomotor precision and design copying at five years of age. Of the children with slightly below average performance in two or more NEPSY II subtests and FSIQ $\geq 70(\mathrm{n}=28)$ at five years of age, seven children $(7 / 28,25 \%)$ received educational support.

NEPSY II scores are presented in Table 3. All mean standard scores of the NEPSY II subtests were lower than the mean value of 10, although in the average range, with the exception of memory for designs (7.0) and design copying (7.2). The highest mean score was in word 
generation (9.6). The memory for designs scores were most commonly significantly below average (34\%) and the scores in design copying were least likely to be on average range (43\%). The word generation scores were least likely to be significantly below average (4\%) and likewise most commonly on average range (76\%).

In the multiple regression analysis, the proportion of variance accounted for by the model was $\omega^{2}=0.12$ for comprehension of instructions, $\omega^{2}=0.09$ for word generation, $\omega^{2}=0.13$ for memory for designs, $\omega^{2}=0.11$ for narrative memory, $\omega^{2}=0.16$ for visuomotor precision, $\omega^{2}$ $=0.14$ for design copying and $\omega^{2}=0.08$ for arrows. Of the background factors, major brain pathology at term equivalent age was associated with poorer scores in comprehension of instructions ( $p<0.01, \beta=-2.11)$, word generation $(p<0.01, \beta=-1.64)$ and design copying $(p<0.01, \beta=-1.62)$. Paternal education of nine to twelve years was associated with poorer scores in memory for designs $(p<0.01, \beta=-1.97)$ and narrative memory $(p<0.01, \beta=$ 1.37). Male sex was associated with lower scores in visuomotor precision $(p<0.01, \beta=-$ 1.40), but also with higher scores in narrative memory $(p<0.01, \beta=1.11)$.

The analyses concerning associations between test scores at five years of age and educational support at eleven years of age (studying on a lower grade, full- or part-time special education) were repeated and adjusted for the background variables that were significantly associated with neuropsychological functions at eleven years of age (gender, paternal education, brain pathology). The associations remained significant between FSIQ and studying on a lower grade, between inhibition and part-time special education, and between comprehension of instructions and studying on a lower grade and full-time special education. 


\section{Discussion}

This longitudinal study of very preterm children showed that both poorer intellectual functioning and weaker neuropsychological functions at five years of age were related to a need for educational support services at eleven years of age. Average neuropsychological performance at eleven years of age was consistently poorer in very preterm children than the normative mean, yet mostly within the average. Risk factors for poorer neuropsychological functions were major brain pathology at term equivalent age, lower paternal education and probably male sex.

General cognitive ability, visuospatial skills, phonological processing, attention and executive functions at six years of age have previously been found to predict academic skills at eleven years of age in children born before 26 gestational weeks ${ }^{11}$. However, it has also been shown that very preterm children at the age of eleven receive more educational support, but their academic skills do not differ from full-term controls when children with significant cognitive impairment are excluded ${ }^{22}$. These results might thus reflect the benefits of support services. In the present study, intellectual functioning and neuropsychological performance at five years of age were shown to be predictive of the need for educational support at eleven years of age. We found that weaker performance at five years of age predicted particularly well a lag in the grade level and a need for full-time special education. Global intellectual functioning as well as language, memory, visuomotor and executive functions at the age of five were all antecedents of the need for later support. Furthermore, also of children with slightly impaired performance at five years of age, one of four received educational support at eleven years of age. Our findings underline the clinical importance of including psychological assessment in the follow-up of very preterm children at five years of age to 
identify those who may need educational support. When impairments or the risk for difficulties are detected, development can be supported through, for example, special education, neuropsychological rehabilitation or occupational therapy.

At eleven years of age, the performance of the very preterm children was poorest in a task assessing visual memory. Visual memory was significantly weaker than average in one third of the very preterm children. Verbal fluency appeared, in turn, to be a relative strength at eleven years of age. At five years of age, the performance was poorest in a task assessing visuomotor precision and strongest in a task assessing verbal memory. It can thus be speculated that some aspects of nonverbal functions might belong to the skills most affected by prematurity. Our findings at eleven years of age are in line with Joseph et al. ${ }^{2}$ who found that neuropsychological functions were consistently below normative expectations in extremely preterm children at ten years of age. Akshoomoff et al. ${ }^{6}$ have in turn reported nonverbal functions being more affected compared to verbal functions in extremely preterm children at the age of ten years.

Our findings highlight that assessment of neuropsychological performance also at eleven years of age is valuable in the developmental follow-up of preterm children in order to identify specific impairments before entering junior high school. It has previously been shown that different groups of preterm children with an approximately average intellectual functioning can have nearly reversed profiles of neuropsychological weaknesses and strengths ${ }^{5}$ and that non-verbal difficulties are not identified in assessment of only intellectual functioning ${ }^{21}$. Neuropsychological deficits, in turn, are related to learning difficulties. For example, mathematical disability has been associated with weaknesses in visual perception 
and attention in extremely preterm children, and reading disability has been linked with poorer naming speed ${ }^{6}$. Neuropsychological assessment provides knowledge about factors behind learning difficulties and about other strengths and weaknesses, which is essential in the planning of appropriate educational support, compensatory strategies and rehabilitation. Failure to identify specific impairments may lead to problems with self-esteem and behavior, and later to inappropriate education choices and marginalization.

Our study showed that very preterm children had poorer neuropsychological performance than term born children both at five and eleven years of age. There are, however, also studies reporting developmental catch-up regarding visuospatial working memory capacity ${ }^{27}$, receptive vocabulary ${ }^{15}$ and some aspects of executive functions ${ }^{28}$ in preterm children. Stålnacke et al. ${ }^{19}$ showed improvement in cognitive abilities between five and 18 years of age in some subgroups of preterm children, while others deteriorated. To this date, long-term developmental outcome cannot be reliably predicted based on risk factors or early developmental assessment, and systematic long-term follow-up of very preterm children is therefore necessary. A number of studies show that preterm children do not catch up with their term-born peers during childhood and adolescence, and that developmental difficulties in preterm children are unlikely to reflect just a developmental delay ${ }^{1,7,13,16,17}$. Therefore, psychological assessment of very preterm children is clinically valuable also at eleven years of age in order to target educational support services. It has been reported that teachers lack knowledge about the consequences of prematurity and many feel inadequately equipped to support prematurely born students ${ }^{29}$, and psychological assessment should be provided with low threshold even in mild difficulties in very preterm children. 
According to a review by Linsell et al. ${ }^{30}$, different factors have an impact on cognitive outcomes at different ages in very preterm or very low birth weight children. Male sex, nonwhite ethnicity, lower parental education and lower birth weight were predictive of global cognitive impairment in children younger than five years. Beyond five years of age, the impact of perinatal factors appeared to diminish and, instead, parental education was shown to influence outcome more. The findings on the role of neonatal brain injury on cognitive development are mixed according to Linsell et al. In our study, the selection of background factors was grounded on previous follow-up studies on intellectual functioning and neuropsychological functions in the PIPARI project ${ }^{4,21}$. In addition to paternal education, major neonatal brain pathology and probably male sex were risk factors for the neuropsychological profile at eleven years of age.

The assessment of precursors of the need for educational support services offers a novel perspective to the need of follow-up. The PIPARI study is a well-designed, prospective longterm follow-up study of a six-year cohort of very preterm children. We chose and limited the set of analyses carefully, but the large amount of statistical analyses may have resulted in some significant associations emerging by chance. However, the associations were to the expected direction except better narrative memory in males. Direct comparison of the neuropsychological profiles at five and eleven years of age was not a focus of this study because different, age-appropriate sets of tests were used in the assessments.

In conclusion, this study highlights the clinical value of psychological assessments at five as well as at eleven years of age in the follow-up of very preterm children. In addition to the assessment of intellectual functioning, neuropsychological functions should be evaluated. 
Even minor deviations in development or learning in preterm children warrant careful attention and early developmental or educational support may also prevent secondary problems. 


\section{Acknowledgements}

The PIPARI Study Group includes, Satu Ekblad, RN; Eeva Ekholm, MD, PhD; Leena Haataja, MD, PhD; Mira Huhtala, MD, PhD; Maija Juntunen, MA; Pentti Kero, MD, PhD; Mari Koivisto, MSc, Riikka Korja, PhD; Katri Lahti, MD; Helena Lapinleimu, MD, PhD; Liisa Lehtonen, MD, PhD; Tuomo Lehtonen, MD; Marika Leppänen, MD, PhD; Annika Lind, PhD; Hanna Manninen, MD; Jonna Maunu, MD, PhD; Petriina Munck PhD; Laura Määttänen, MD; Pekka Niemi, PhD; Anna Nyman, MA; Pertti Palo, MD, PhD; Riitta Parkkola, MD, PhD; Päivi Rautava, MD, PhD; Katriina Saarinen, Physiotherapist; Susanna Salomäki, MA; Virva Saunavaara, PhD; Sirkku Setänen, MD, PhD; Matti Sillanpää, MD, PhD; Suvi Stolt, PhD; Päivi Tuomikoski-Koiranen, RN; Timo Tuovinen, BA; Karoliina Uusitalo, MD; Milla Ylijoki, MD, PhD.

Disclosure of interest: The authors have no conflict of interest to report. 


\section{References}

(1) Anderson PJ. Neuropsychological outcomes of children born very preterm. Semin Fetal Neonatal Med 2014;19:90-6.

(2) Joseph RM, O'Shea TM, Allred EN, Heeren T, Hirtz D, Jara H, et al. Neurocognitive and Academic Outcomes at Age 10 Years of Extremely Preterm Newborns. Pediatrics 2016;137:e20154343.

(3) Kallankari H, Kaukola T, Olsen P, Ojaniemi M, Hallman M. Very preterm birth and foetal growth restriction are associated with specific cognitive deficits in children attending mainstream school. Acta Paediatr 2015;104:84-90.

(4) Lind A, Korkman M, Lehtonen L, Lapinleimu H, Parkkola R, Matomaki J, et al. Cognitive and neuropsychological outcomes at 5 years of age in preterm children born in the 2000s. Dev Med Child Neurol 2011;53:256-62.

(5) Lundequist A, Bohm B, Smedler AC. Individual neuropsychological profiles at age 5(1/2) years in children born preterm in relation to medical risk factors. Child Neuropsychol 2013;19:313-31.

(6) Akshoomoff N, Joseph RM, Taylor HG, Allred EN, Heeren T, O'Shea TM, et al. Academic Achievement Deficits and Their Neuropsychological Correlates in Children Born Extremely Preterm. J Dev Behav Pediatr 2017;38:627-37.

(7) Brydges CR, Landes JK, Reid CL, Campbell C, French N, Anderson M. Cognitive outcomes in children and adolescents born very preterm: a meta-analysis. Dev Med Child Neurol 2018;60:452-68. 
(8) Doyle LW, Davis PG, Schmidt B, Anderson PJ. Cognitive outcome at 24 months is more predictive than at 18 months for IQ at 8-9 years in extremely low birth weight children. Early Hum Dev 2012;88:95-8.

(9) Halbwachs M, Muller JB, Nguyen The Tich S, Gascoin G, Chauty-Frondas A, Branger B, et al. Predictive value of the parent-completed ASQ for school difficulties in preterm-born children <35 weeks' GA at five years of age. Neonatology 2014;106:311-16.

(10) Hornman J, de Winter AF, Kerstjens JM, Bos AF, Reijneveld SA. Stability of Developmental Problems after School Entry of Moderately-Late Preterm and Early PretermBorn Children. J Pediatr 2017;187:73-9.

(11) Johnson S, Wolke D, Hennessy E, Marlow N. Educational outcomes in extremely preterm children: neuropsychological correlates and predictors of attainment. Dev Neuropsychol 2011;36:74-95.

(12) Leversen KT, Sommerfelt K, Elgen IB, Eide GE, Irgens LM, Juliusson PB, et al. Prediction of outcome at 5 years from assessments at 2 years among extremely preterm children: a Norwegian national cohort study. Acta Paediatr 2012;101:264-70.

(13) Linsell L, Johnson S, Wolke D, O'Reilly H, Morris JK, Kurinczuk JJ, et al. Cognitive trajectories from infancy to early adulthood following birth before 26 weeks of gestation: a prospective, population-based cohort study. Arch Dis Child 2018;103:363-70.

(14) Luttikhuizen dos Santos ES, de Kieviet JF, Konigs M, van Elburg RM, Oosterlaan J. Predictive value of the Bayley scales of infant development on development of very preterm/very low birth weight children: a meta-analysis. Early Hum Dev 2013;89:487-96. 
(15) Luu TM, Vohr BR, Allan W, Schneider KC, Ment LR. Evidence for catch-up in cognition and receptive vocabulary among adolescents born very preterm. Pediatrics. 2011;128:313-22.

(16) Mangin KS, Horwood LJ, Woodward LJ. Cognitive Development Trajectories of Very Preterm and Typically Developing Children. Child Dev 2017;88:282-98.

(17) Nguyen TN, Spencer-Smith M, Zannino D, Burnett A, Scratch SE, Pascoe L, et al. Developmental Trajectory of Language From 2 to 13 Years in Children Born Very Preterm. Pediatrics 2018;141:e20172831.

(18) Potharst ES, Houtzager BA, van Sonderen L, Tamminga P, Kok JH, Last BF, et al. Prediction of cognitive abilities at the age of 5 years using developmental follow-up assessments at the age of 2 and 3 years in very preterm children. Dev Med Child Neurol 2012;54:240-46.

(19) Stalnacke J, Lundequist A, Bohm B, Forssberg H, Smedler AC. Individual cognitive patterns and developmental trajectories after preterm birth. Child Neuropsychol 2015;21:64867.

(20) Munck P, Niemi P, Lapinleimu H, Lehtonen L, Haataja L, PIPARI Study Group. Stability of cognitive outcome from 2 to 5 years of age in very low birth weight children. Pediatrics 2012;129:503-8.

(21) Nyman A, Korhonen T, Munck P, Parkkola R, Lehtonen L, Haataja L. Factors affecting the cognitive profile of 11-year-old children born very preterm. Pediatr Res 2017;82:324-32. 
(22) Nyman A, Korhonen T, Lehtonen L, Haataja L on behalf of the PIPARI Study Group. School performance is age-appropriate with support services in very preterm children at 11 years of age. Acta Paediatrica 2019 [Epub ahead of print]

(23) Wechsler D. Wechsler Preschool and Primary Scale of Intelligence-Revised (Finnish translation). Helsinki: Psykologien Kustannus, 1995.

(24) Korkman M, Kirk U, Kemp SL. NEPSY II, 2nd edn. San Antonio, TX: PsychCorp/ Pearson Clinical Assessment, 2007.

(25) Korkman M, Kirk U, Kemp SL. NEPSY II. Helsinki: Psykologien Kustannus, 2008.

(26) Lind A, Haataja L, Rautava L, Valiaho A, Lehtonen L, Lapinleimu H, et al. Relations between brain volumes, neuropsychological assessment and parental questionnaire in prematurely born children. Eur Child Adolesc Psychiatry 2010;19:407-17.

(27) Murner-Lavanchy I, Ritter BC, Spencer-Smith MM, Perrig WJ, Schroth G, Steinlin M, et al. Visuospatial working memory in very preterm and term born children-impact of age and performance. Dev Cogn Neurosci 2014;9:106-16.

(28) Réveillon M, Hüppi PS, Barisnikov K. Inhibition difficulties in preterm children: Developmental delay or persistent deficit? Child Neuropsychol 2018;24:734-62.

(29) Johnson S, Gilmore C, Gallimore I, Jaekel J, Wolke D. The long-term consequences of preterm birth: what do teachers know? Dev Med Child Neurol 2015;57:571-7.

(30) Linsell L, Malouf R, Morris J, Kurinczuk JJ, Marlow N. Prognostic Factors for Poor Cognitive Development in Children Born Very Preterm or With Very Low Birth Weight: A Systematic Review. JAMA Pediatr 2015;169:1162-72. 
Table 1. Background data on the very preterm children $(n=167)$ and the drop out children $(n=52)$.

Very preterm children

Maternal education

$<9$ years / 9-12 years / > 12 years, $\mathrm{n}(\%)$

Paternal education

$<9$ years / 9-12 years / > 12 years, $\mathrm{n}(\%)$

Boys/girls, n (\%)

Gestational age, weeks, mean (SD) min-max

Birth weight, g, mean (SD) min-max

Birth weight $\mathrm{z}$ score, mean (SD) min-max

Postnatal corticosteroids, $\mathrm{n}(\%)$

Chronic lung disease, sepsis or meningitis and/or intestinal perforation, $\mathrm{n}(\%)$

Brain magnetic resonance imaging findings at term equivalent age

normal findings, $\mathrm{n}(\%)$

minor pathology, $\mathrm{n}(\%)$
19 (11) / 41 (25) / 105 (63)

$14(8) / 95(57) / 54(32)$
$92(55) / 75(45)$
$28.9(2.7) 23.0-35.9$
$1116(311) 400-2120$
$-1.4(1.5)-4.9-3.4$
$22(13)$

$52(31)$
$93(56)$

$32(62)$

$26(16)$

8 (15)
10 (19) / 15 (29) / 23 (44)

0.11

$12(23)$

0.26

0.59

P-value

$\begin{array}{ll}8(15) / 25(48) / 14(27) & 0.25 \\ 32(62) / 20(38) & 0.41 \\ 29.4(2.9) 23.7-34.9 & 0.30 \\ 1239(369) 590-2025 & 0.02 \\ -1.2(1.4)-4.3-2.2 & 0.36 \\ 7(13) & 0.96\end{array}$

0.96 
In the very preterm group data on maternal education was missing for two children, data on paternal education for four children and data on magnetic resonance imaging for four children. In the drop out group data on maternal education was missing for four children, data on paternal education for five children and data on magnetic resonance imaging for two children. 
Table 2a. Full scale intelligence quotient and NEPSY II standard scores of the very preterm children $(n=150)$ at five years of age, and the percentages of the NEPSY II standard scores considered as average $(\geq 8)$, slightly below average (6-7) and significantly below average ( $\leq$ $5)$.

Mean (SD) $\quad \geq 8 \quad 6-7 \quad \leq 5$

Full-scale intelligence quotient

$101.5(17.4)$

Visual attention

$8.6(2.4)$

$65 \%$

$17 \%$

$18 \%$

Auditory attention

$8.8(3.2)$

$63 \%$

$17 \%$

$21 \%$

Inhibition

$8.7(3.2)$

$53 \%$

$21 \%$

$25 \%$

Speeded naming

$8.9(2.8)$

$71 \%$

$13 \%$

$16 \%$

Comprehension of instructions

$9.3(3.0)$

$71 \%$

$17 \%$

$12 \%$

Phonological processing

$8.9(2.4)$

$69 \%$

$26 \%$

$5 \%$

Memory for designs

$8.3(2.9)$

$61 \%$

$17 \%$

$22 \%$

Narrative memory

$9.8(2.4)$

$77 \%$

$13 \%$

$9 \%$

Word list interference

$8.4(3.9)$

$57 \%$

$17 \%$

$26 \%$

Visuomotor precision

$8.1(3.3)$

$47 \%$

$35 \%$

$17 \%$

Design copying

$8.6(3.2)$

$62 \%$

$19 \%$

$19 \%$

Missing data: Full-scale intelligence quotient $=7$; Visual attention $=8 ;$ Auditory attention $=17$; Inhibition $=13 ;$ Speeded naming $=13$; Comprehension of instructions $=3$; Phonological processing $=1$; Memory for designs $=9$; Narrative memory $=10$; Word list interference $=12 ;$ Visuomotor precision $=3$; Design copying $=17$ 
Table 2b. Univariate associations, odds ratio (95\% confidence interval) and p-value, between test scores at five years of age and the need for educational support at eleven years of age in very preterm children $(n=150)$.

Grade/grades below

own age group $(\mathrm{n}=32)$
Full-time special

education $(\mathrm{n}=20)$
Part-time special

education $(n=29)$
Personal assistant

$(\mathrm{n}=3)$

$\begin{array}{lllll}\text { Full-scale intelligence quotient } & 0.94(0.91-0.97)<0.01^{*} & 0.95(0.92-0.98)<0.01^{*} & 0.97(0.95-0.99) 0.01 & 0.92(0.86-0.99) 0.03 \\ \text { Visual attention } & 0.93(0.78-1.10) 0.40 & 1.07(0.86-1.34) 0.54 & 0.91(0.76-1.08) 0.27 & 2.14(0.69-6.68) 0.19 \\ \text { Auditory attention } & 0.87(0.75-1.00) 0.05 & 0.87(0.73-1.02) 0.09 & 0.87(0.76-1.00) 0.05 & 0.67(0.38-1.20) 0.18 \\ \text { Inhibition } & 0.85(0.73-0.98) 0.02 & 0.77(0.62-0.95) 0.02 & 0.73(0.61-0.87)<0.01 * & 0.80(0.49-1.30) 0.36 \\ \text { Speeded naming } & 0.86(0.73-1.02) 0.08 & 0.81(0.65-0.99) 0.04 & 0.95(0.82-1.11) 0.52 & 0.80(0.43-1.50) 0.48 \\ \text { Comprehension of instructions } & 0.73(0.62-0.85)<0.01^{*} & 0.67(0.54-0.82)<0.01^{*} & 0.89(0.77-1.02) 0.09 & 0.56(0.37-0.97) 0.04 \\ \text { Phonological processing } & 0.84(0.71-0.99) 0.04 & 0.92(0.76-1.12) 0.43 & 0.81(0.68-0.97) 0.02 & 0.81(0.52-1.26) 0.35 \\ \text { Memory for designs } & 0.80(0.69-0.92)<0.01^{*} & 0.80(0.67-0.95) 0.01 & 0.86(0.74-0.99) 0.04 & 0.69(0.45-1.07) 0.10 \\ \text { Narrative memory } & 0.88(0.73-1.06) 0.17 & 0.94(0.75-1.18) 0.59 & 0.97(0.81-1.15) 0.71 & 1.03(0.46-2.29) 0.95 \\ \text { Word list interference } & 0.86(0.77-0.96) 0.01 & 0.92(0.80-1.05) 0.22 & 0.96(0.87-1.07) 0.49 & 0.92(0.57-1.48) 0.73 \\ \text { Visuomotor precision } & 0.74(0.61-0.91)<0.01^{*} & 0.72(0.56-0.93) 0.01 & 1.04(0.92-1.17) 0.58 & 0.60(0.26-1.39) 0.24 \\ \text { Design copying } & 0.88(0.77-1.00) 0.06 & 0.76(0.65-0.90)<0.01^{*} & 0.94(0.82-1.08) 0.38 & 0.75(0.52-1.10) 0.14\end{array}$

$* p<0.01$

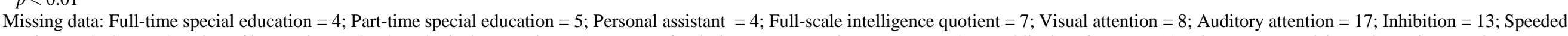

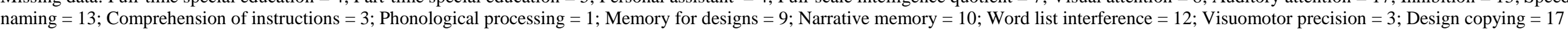


Table 3. NEPSY II standard scores of the very preterm children $(n=167)$ at eleven years of age, and the percentages of the standard scores considered as average $(\geq 8)$, slightly below average (6-7) and significantly below average $(\leq 5)$.

\begin{tabular}{lllll}
\hline & Mean (SD) & $\geq 8$ & $6-7$ & $\leq 5$ \\
& & & & \\
\hline Comprehension of instructions & $8.0(3.2)$ & $68 \%$ & $9 \%$ & $23 \%$ \\
Word generation & $9.6(2.7)$ & $76 \%$ & $20 \%$ & $4 \%$ \\
Memory for designs & $7.0(3.5)$ & $47 \%$ & $19 \%$ & $34 \%$ \\
Narrative memory & $8.5(2.4)$ & $65 \%$ & $25 \%$ & $10 \%$ \\
Visuomotor precision & $8.2(2.3)$ & $62 \%$ & $25 \%$ & $13 \%$ \\
Design copying & $7.2(2.3)$ & $43 \%$ & $35 \%$ & $22 \%$ \\
Arrows & $8.3(3.5)$ & $59 \%$ & $25 \%$ & $17 \%$ \\
& & & & \\
\hline
\end{tabular}

Data on design copying was missing for two children. 